\title{
Developing healthcare systems to support exercise: exercise as the fifth vital sign
}

\author{
Robert Sallis
}

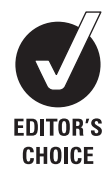

The importance of physical activity to health and wellness has been established incontrovertibly. There is a linear relationship between physical activity and health-those who maintain an active and fit way of life live longer, healthier lives. ${ }^{1}$ Those who are unfit and sedentary very predictably develop chronic diseases prematurely and die at a younger age. ${ }^{1}$ This association between activity level and health status exists in various major subgroups of the population. It doesn't matter whether you study men or women, various ethnic groups, diverse nations, children or the aged, the results are always the same: people who are active and fit live longer, healthier lives. This is no longer news.

\section{ASSESSING PHYSICAL ACTIVITY LEVELS AT EVERY CLINICAL CONSULT}

Exercise, therefore, is medicine that every patient needs to take. Its tremendous and proven clinical benefits should not be denied any patient. Especially in patients who have chronic diseases like diabetes, heart disease and hypertension to list just three. Likewise, anyone who is at risk for these chronic diseases should consider exercise an essential vaccine to greatly lower risk of illness and almost certainly extend life. The ready availability of this powerful therapy means that a patient's exercise habits should be assessed at every clinical visit and used by the clinician in the management of every patient. ${ }^{2}$

Unfortunately, the medical community has resisted attempts to utilise exercise as a medication, believing that doctors prescribing exercise has no effect on our patient's behaviour. That just does not make sense! On a daily basis, we physicians convince our patients to undertake therapy that is far more time-consuming, uncomfortable and even dangerous, by explaining how

Correspondence to Dr Robert Sallis, Department of Family Medicine, Kaiser Permanente Medical Center, 9961 Sierra Avenue, Fontana, CA, 92336, USA; robert.e.sallis@kp.org important that therapy is to treating or preventing disease. Patients are always very resistant to taking insulin shots, to chemotherapy treatments and to invasive surgical procedures, yet every day they carry out doctor's orders for such therapy in the belief they will be helped and because we have a system set up to administer these treatments. If a similar system were in place to support physical activity as a therapy, I have no doubt patients would be more likely to consider exercise as a medication and take it as directed.

An essential first step in our healthcare systems needed to get patients more active is to query them on their exercise habits. Every healthcare provider should obtain an 'exercise vital sign' (or 'physical activity vital sign') on every patient they see. There is no better indicator of a person's health and likely longevity than the min/ week of activity they engage in. I would suggest it makes more sense to stop measuring blood pressure at every visit than to ignore our patient's exercise habits, because we know that being sedentary is a bigger risk factor for mortality than mild to moderate levels of hypertension. ${ }^{3}$ Since few would agree to quit measuring blood pressure as a vital sign, why would we continue to ignore measuring our patient's physical activity levels?

\section{KAISER PERMANENTE EXPERIENCE: TWO QUESTIONS, 1 MINUTE!}

At Kaiser Permanente in California, we use a simple method for taking an 'Exercise Vital Sign' on every patient, at every visit. During each patient intake, when traditional vital signs are checked, the medical assistant asks the patient two questions. The first is 'on average, how many days/ week do you engage in moderate or greater physical activity (like a brisk walk)?' The second question is 'on those days, how many minutes do you engage in activity at this level?' Our computerised medical record then multiplies these two measures to arrive at the average $\mathrm{min} / \mathrm{week}$ of moderate or greater physical activity reported by each patient. This information is then displayed in the vital sign header of the computerised chart so a physician or other healthcare provider can review the reported activity level on each patient.

I will acknowledge the accuracy of selfreported exercise is often low, but would argue this does not diminish the importance of the exercise vital sign. Don't medical providers have an ethical obligation to ask patients about their physical activity habits and inform them of the dangers of being inactive? At that point, patients can at least make an informed decision about how active they choose to be. Whether asking patients about activity level actually changes their behaviour is in some ways a moot point, because at minimum they should be informed. Physicians can be effective in promoting behaviour change ${ }^{4}$; one example is physicians' contribution to reducing smoking rates from about $50 \%$ to close to $20 \% .{ }^{5}$ As physicians, we need to take responsibility for contributing to the socioecological influences ${ }^{6}$ that address the public health problem of physical inactivity. ${ }^{1}$

\section{PRACTICAL THRESHOLDS FOR INITIATING TREATMENT STAT! 150 MIN/WEEK FOR ADULTS}

Adultswhoengageinless than $150 \mathrm{~min} /$ week and children doing less than $420 \mathrm{~min} /$ week should be flagged as not meeting the recommended amounts of physical activity to improve their health. They should be advised on the importance of being more active and ideally offered counselling and support to help them increase their exercise. I have found that patients are most likely to follow an exercise prescription when I connect it to disease they are currently dealing with. Explaining how regular exercise can improve their blood pressure or lower blood sugar so that they might not require insulin is often key to getting patients to follow my exercise advice.

For patients who are really struggling to get more active or who suffer with a disability that makes it difficult for them to just get out and walk, I have found that referral to a fitness professional can be extremely helpful. I am often frustrated that fitness experts are not readily available to my patients. To that end, we need to better link the fitness industry with the healthcare industry. Why do these groups operate so separately? When I have a patient who desperately needs to engage in regular physical activity to improve their health, why can't I refer them to a fitness professional trained to get and keep people active? I am convinced this would do many of my patients more good than 
referral to a medical or surgical subspecialist. Why can I, as a family physician, refer my obese patient to a bariatric surgeon, but not to a fitness professional as part of the healthcare team? The existing system does not make sense!

\section{PRACTICAL STEPS IN YOUR OFFICE}

There is a long way to go to develop a healthcare system that supports getting patients more active. As the burden of chronic disease associated with inactivity is growing at a staggering rate, healthcare providers can no longer ignore the growing evidence connecting physical inactivity and poor health. Clinicians have a duty to assess the exercise habits of every patient and make sure the patient understands the health risks associated with inactivity. We should also explore practical ways to help patients overcome barriers to carrying out an exercise prescription, such as referral to a fitness professional. As a clinician, have you met with local fitness professionals to
To listen to a free podcast of Dr Sallis recorded in 2010, follow this link (http:// podcasts.bmj.com/bjsm/2010/06/01/). A related podcast to reference 1 in this article is by Professor Steven Blair (http:// podcasts.bmj.com/bjsm/2010/08/16/). For exercise is medicine tips, add BJSM as a Twitter feeds (@BJSM_BMJ).

see how you can cooperate with them? Have you invited them to give a talk at your practice? Are there forms to facilitate referral? Do you have the 'Exercise is Medicine' website information handy? $B J S M$ encourages your suggestions of practical ways to get patients movinge-mail the editorial office to get your thoughts on the BJSM Blog. Exercise is medicine, and we need to do all we can to get our patients to take it!

Competing interests None.

Provenance and peer review Commissioned; not externally peer reviewed.
Accepted 30 December 2010

Published Online First 3 February 2011

Br J Sports Med 2011;45:473-474.

doi:10.1136/bjsm.2010.083469

\section{REFERENCES}

1. Blair SN. Physical inactivity: the biggest public health problem of the 21 st century. Br J Sports Med 2009;43:1-2.

2. Sallis RE. Exercise is medicine and physicians need to prescribe it! Br J Sports Med 2009;43:3-4.

3. Physical Activity Guidelines Advisory Committee. Physical Activity Guidelines Advisory Committee Report 2008. Washington, DC: Physical Activity Guidelines Advisory Committee, 2008.

4. Meyer C, Ulbricht S, Baumeister SE, et al. Proactive interventions for smoking cessation in general medical practice: a quasi-randomized controlled trial to examine the efficacy of computer-tailored letters and physician-delivered brief advice. Addiction 2008; 103:294-304.

5. Prokhorov AV, Hudmon KS, Marani S, et al. Engaging physicians and pharmacists in providing smoking cessation counseling. Arch Intern Med 2010;170:1640-6.

6. Stokols D. Translating social ecological theory into guidelines for community health promotion. Am J Health Promot 1996;10:282-98. 